\title{
Perspective
}

PERSPECTIVE Actualité en histoire de l'art

$2 \mid 2021$

Habiter

\section{Écrire une histoire de l'architecture de la vie privée}

Un entretien avec Monique Eleb, par Estelle Thibault

Monique Eleb et Estelle Thibault

\section{OpenEdition}

Journals

Édition électronique

URL : https://journals.openedition.org/perspective/25185

DOI : $10.4000 /$ perspective.25185

ISSN : 2269-7721

Éditeur

Institut national d'histoire de l'art

Édition imprimée

Date de publication : 30 décembre 2021

Pagination : 111-130

ISBN : 978-2-917902-92-9

ISSN : $1777-7852$

Référence électronique

Monique Eleb et Estelle Thibault, «Écrire une histoire de l'architecture de la vie privée », Perspective [En ligne], 2 | 2021, mis en ligne le 30 juin 2022, consulté le 13 février 2022. URL : http://

journals.openedition.org/perspective/25185; DOI : https://doi.org/10.4000/perspective.25185 


\title{
Écrire une histoire de l'architecture de la vie privée
}

\author{
Un entretien avec Monique Eleb, \\ par Estelle Thibault
}

Cet entretien avec Monique Eleb, psychologue et sociologue, s'est déroulé à Paris en mai 2021 dans son appartement parisien. Il a été l'occasion de revenir sur la trajectoire d'une chercheuse qui a consacré l'essentiel de ses travaux à l'analyse de l'évolution de l'habitation en France, couvrant une très large période, du début du XVII ${ }^{\mathrm{e}}$ siècle à la production la plus contemporaine. La discussion éclaire la genèse du projet en deux volumes de l'ouvrage Architectures de la vie privée (Maisons et mentalités, XVII ${ }^{e}$-XIX ${ }^{e}$ siècles, paru en 1989, puis L'invention de l'habitation moderne, Paris 1880-1914, publié en 1995). Un troisième volume, consacré à l'entre-deux-guerres, est en cours de rédaction. Le parcours de Monique Eleb se singularise par son inclination pour l'interdisciplinarité, depuis sa formation initiale en psychologie et sociologie à la Sorbonne dans les années 1960, en passant par l'expérience de la recherche à l'Institut de l'environnement, où les sciences humaines côtoyaient les pratiques de conception allant du design à l'urbanisme, jusqu'aux écoles d'architecture où elle développa ses enseignements et de nombreux travaux portant sur l'analyse des logements contemporains.

Adopter une démarche historique n'avait rien d'évident pour quelqu'un dont la thèse proposait une " analyse psychosociale clinique " des interventions par lesquelles des habitants s'approprient leur espace domestique (ELEB-VIDAL, 1980). En effet, la chercheuse s'intéressait initialement à la manière dont la construction de l'identité s'inscrit dans les transformations des logements. C'est la volonté de mettre en perspective ces phénomènes qui lui a rendu nécessaire le déploiement d'une enquête rétrospective sur l'architecture de l'habitation, interrogeant l'émergence des notions d'" intimité ", de "public " et de " privé " dans la conception des espaces. L'originalité de la démarche résidait dans le fait d'aborder l'histoire des mentalités par l'examen des dispositifs spatiaux, tout particulièrement des systèmes de distribution et de composition qui organisent les rapports interpersonnels, entre hommes et femmes, adultes et enfants, maîtres et domestiques.

L'entretien fait une large place à la question des méthodes hybrides et de la diversité des sources utiles pour une telle enquête, mobilisant des documents textuels (traités d'architecture ou de savoir-vivre, littérature, dictionnaires...) et un vaste ensemble de 
documents graphiques (plans, tableaux, gravures, etc.). La méthode de lecture des plans, construite au contact des architectes, fut croisée avec l'interprétation des autres sources et enrichie par les apports des sciences humaines, en mobilisant un vaste ensemble d'outils et de concepts. L'entretien revient aussi sur les façons d'étudier les évolutions récentes des logements et sur la nécessité d'inscrire dans une perspective de longue durée ce qui est aujourd'hui présenté comme innovant ou expérimental. Enfin, l'intérêt des travaux de Monique Eleb réside aussi dans son implication dans l'analyse critique de la production contemporaine, dans la formation des architectes ainsi qu'au sein d'équipes de conception pluridisciplinaires.

[Estelle Thibault]

- Estelle Thibault. «Je n'ai jamais choisi de faire de I'histoire» (ELEB, 2002, p. 137). Cette phrase ouvrait un article dans lequel vous reveniez sur les méthodes d'analyse que vous avez déployées dans vos différents travaux sur l'évolution de l'habitation en France. Quel a été votre parcours ? Comment votre formation initiale de psychologue vous a-t-elle conduite à vous intéresser à l'architecture?

- Monique Eleb. J'ai d'abord fait des études de psychologie et de sociologie, dans la perspective de devenir psychanalyste. Il s'agissait au début essentiellement d'une formation en sciences dites dures (psychologie expérimentale, beaucoup de statistiques et de méthode). À l'époque où j'ai commencé mes études, au milieu des années 1960, il y avait des cours communs aux cursus de psychologie et de sociologie pendant les deux premières années, mais les orientations étaient très différentes. Toutes les disciplines m'intéressaient, l'anthropologie notamment. Ma sœur ${ }^{1}$ étudiait alors l'architecture à l'École des beaux-arts et je fréquentais un certain nombre d'architectes qui me posaient des questions sur la psychologie de la forme. Je m’orientais vers la psychanalyse mais j'étais également passionnée par la psychologie de la perception et la psychophysiologie. Au moment de ma maîtrise, par l'intermédiaire d'une assistante de l'université, j'ai eu l'opportunité de terminer une bibliographie concernant les relations entre sciences humaines et architecture. J'ai ainsi travaillé tout l'été pour l'Institut de l'environnement. Cet établissement pilote venait d'être créé par le ministère de la Culture, en 1969, afin de tenter de faire émerger une pédagogie alternative, interdisciplinaire, sur le modèle de la Hochschule für Gestaltung d'Ulm. Alors dirigé par l'architecte Claude Schnaidt, qui en revenait, l'Institut de l'environnement était une tentative de pluridisciplinarité. L'équipe comprenait par exemple l'urbaniste Jacques Allégret, fondateur de l'atelier d'Architecture et d'Urbanisme (AUA) qui mettait en œuvre une approche pluridisciplinaire, le sociologue Antoine Haumont ou encore le mathématicien Jean Zeitoun. Comme l'a décrit l'ouvrage de Tony Côme, il s'agissait d'un lieu de formation post-diplôme qui accueillait des étudiants très exigeants et très politisés ${ }^{2}$. L'équipe enseignante rassemblait des praticiens, notamment architectes et urbanistes, et des chercheurs de diverses disciplines, des mathématiques aux diverses branches des sciences humaines. La bibliographie en question a eu beaucoup de succès ${ }^{3}$, ce qui était un signe de l'appétit de ce milieu pour les sciences humaines, à la grande époque de Roland Barthes et de Michel Foucault. Le psychanalyste Christian Gaillard, qui devint plus tard professeur de psychanalyse de l'art à l'École des beaux-arts de Paris, dirigeait alors l'unique centre de recherche, en sciences humaines, et m'a proposé de rester. Nous avons été rejoints par d'autres chercheurs comme l'économiste Thérèse Evette ainsi que par divers architectes : Philippe Nick puis Bruno Fortier, Jean-Louis Cohen, David Élalouf, Pierre Clément ont été recrutés à l'Institut. La constitution du fonds du centre de documentation, 
dont était chargé ce dernier, fut essentielle. Pendant sept ans j'ai énormément lu, aussi bien des ouvrages de sciences humaines que des traités et théories de l'architecture. J'y ai acquis une culture fondamentale, même si mon arrivée dans ce lieu fut initialement assez fortuite.

- Estelle Thibault. Le goût de l'interdisciplinarité est-il né de cette expérience à l'Institut de l'environnement? Quelles ont alors été les rencontres et les lectures marquantes?

- Monique Eleb. Dans un tel contexte, la recherche se présentait comme une perspective plus enthousiasmante pour moi que la pratique de la psychanalyse. Je me formais à la fois d'un point de vue théorique, en menant des entretiens et en écrivant des articles. J'ai par exemple participé à une enquête sur l'identité des architectes ${ }^{4}$ et travaillé au premier numéro des Cahiers pédagogiques de l'Institut de l'environnement, intitulé Psychologie et espace. Nous avions le temps et les instruments, une imprimerie intégrée... Parallèlement était créé en 1972 le comité de la Recherche et du développement en architecture (CORDA).

Avec Christian Gaillard, nous nous sommes demandé comment travailler en liaison avec les unités pédagogiques d'architecture, qui s'étaient créées après 1968 suite à l'éclatement de la section architecture à l'École des beaux-arts. Nous avons organisé le séminaire « Sciences humaines et architecture » de façon à réfléchir sur l'enseignement et effectué une enquête qui a été publiée sous le titre Espace des sciences humaines (GAILLARD, TEBOUL, VIDAL, 1973). Cet ouvrage rassemblait des entretiens que j'ai conduits avec Gustave Massiah, économiste, Christelle Robin, psychologue ${ }^{5}$, Ginette Baty-Tornikian, anthropologue, Suzanne Paré, géographe, François Lautier, Bernard Haumont et Claire Thierry, sociologues urbains... Pendant ce temps j'ai commencé à enseigner à l'unité pédagogique d'Architecture $n^{\circ} 1^{6}$ avec d'autres sociologues et avec des architectes. On m'a d'abord demandé d'enseigner la psychologie de la forme et des questions relatives au corps dans l'espace, des thèmes que je maîtrisais puisqu'ils faisaient partie de ma formation initiale.

La question de savoir quelle place donner aux sciences humaines dans la formation des architectes avait été posée quand Max Querrien, directeur de l'Architecture au ministère des Affaires culturelles, reçut la mission de réformer l'enseignement de l'architecture et mit en place, en 1966, une commission, avec notamment le sociologue Henri Lefebvre qui présida en 1967 celle de sciences humaines. Nos interviews collectées pour Espace des sciences humaines montraient qu'un clivage existait entre, d'un côté, des enseignants qui considéraient avoir à se former à l'architecture et, de l'autre, ceux qui pensaient pouvoir enseigner comme à l'Université. J'étais plutôt du côté des premiers.

En 1976 je me suis engagée dans ma thèse de doctorat (ELEB-VIDAL, 1980), me tournant alors vers I'habitat, qui n'était pas mon idée première. Je me suis demandé quelles représentations avaient les architectes de la vie quotidienne et de l'architecture domestique. À l'époque étaient formulées dans la presse des critiques fondamentales sur les grands ensembles, accusés de rendre malades leurs habitants. Michèle Huguet, dans Les Femmes dans les grands ensembles ${ }^{7}$, avait pris la question de front. Pour répondre, elle avait comparé les demandes d'aide psychologique dans un tissu pavillonnaire à celles émanant des habitants des grands ensembles. Elle mettait en évidence une différence entre, d'un côté, les crises qui prédominaient chez des habitantes de grands ensembles en moyenne plus jeunes et, de l'autre, des maladies chroniques installées, dans les quartiers pavillonnaires. Son ouvrage éclairait également divers aspects relatifs aux représentations du phénomène des grands ensembles.

Si l'enquête bibliographique "Sciences humaines et architecture » m'avait fait découvrir de nombreux travaux, elle révélait aussi les manques. Paul-Henry et Marie-José Chombart de Lauwe travaillaient par enquêtes, sur le réel, avec le groupe d'ethnologie sociale ${ }^{8}$. Colette Pétonnet avait mené une enquête anthropologique sur une cité de transit de la région 
parisienne ${ }^{9}$. Nicole et Antoine Haumont, avec Marie-Geneviève et Henri Raymond, plus proches de la sociologie d'Henri Lefebvre, avaient publié $L^{\prime} H a b i t a t$ pavillonnaire ${ }^{10}$. Hormis ces travaux, peu de chercheurs s'intéressaient aux problématiques liées à l'habitat.

De mon côté, la question qui me préoccupait pour ma thèse de doctorat était la suivante : pourquoi transforme-t-on son logement? Dans cette perspective, je me lançai dans l'analyse de I'habitat, qui a par la suite organisé toute ma vie... J'explorai ainsi un vaste champ de références. Je m'intéressais à la question de l'identité en même temps que je lisais les théories sur la distribution et les écrits des architectes ( $\mathrm{d}^{\prime}$ Andrea Palladio à Aldo Rossi ${ }^{11}$ ). La lecture de I'Esquisse d'une théorie de la pratique de Pierre Bourdieu ${ }^{12}$, les réflexions de Marcel Mauss sur les " techniques du corps ${ }^{13}$ » ont été inspirantes. De même, celles d'Erving Goffman ${ }^{14}$ sur l'interaction m'ont été utiles pour analyser les comportements dans la vie quotidienne.

- Estelle Thibault. Pour autant, l'interrogation sur les raisons motivant les transformations des logements concernait prioritairement l'architecture contemporaine. Comment en êtes-vous venue à entreprendre un travail historique ?

- Monique Eleb. Quand j'ai rédigé mon habilitation à diriger des recherches, je me suis interrogée sur l'unité de mon parcours et sur les questions qui avaient émergé dans le sillage de ma thèse de doctorat (ELEB-VIDAL, 1980). Celle-ci, réalisée entre 1976 et 1980, portait sur deux tours d'un grand ensemble de Bagnolet, I'une d'habitation à loyer modéré (HLM), l'autre relevant du 1\% patronal avec beaucoup de personnel de la Société nationale des chemins de fer français. Dans ces tours habitaient aussi bien des grandes familles émigrées que des artistes logés dans les ateliers des derniers étages. Cette mixité sociale résultait d'une action volontariste de la part de l'Office des HLM. Les habitants y avaient opéré un nombre impressionnant de transformations. Sur les dix-sept étages des deux tours, aucun appartement ne se ressemblait, certains avaient fermé la cuisine, modifié le séjour, d'autres avaient descendu des murs... Je souhaitais comprendre ce qui avait motivé ces changements et ce que cela signifiait, d'un point de vue psychologique et sociologique. S'agissait-il de laisser sa marque ? Les habitants me parlaient tantôt d'intimité, tantôt de leur désir d'être moderne. Comment les questions d'identité s'inscrivaient-elles dans ses transformations ? Le titre de la thèse, "Se construire et habiter ", reflète cette problématique. Mais à la fin de ce travail, j'avais aussi l'intuition que ces phénomènes ne pouvaient être appréhendés hors de l'histoire du logement et des mœurs. J'ai cru à l'époque pouvoir trouver aisément des livres pour saisir les traditions dans lesquelles s'inscrivaient les organisations spatiales observées. Je ne comprendrais que plus tard, par exemple, que la petite cuisine laboratoire avec son cellier, éclairée en second jour au fond de la salle à manger était redevable d'Auguste Perret et que l'ouverture des espaces les uns sur les autres révélait une influence corbuséenne.

Jean-Louis Cohen, qui était alors mon collègue, venait d'être nommé au Secrétariat de la recherche architecturale (SRA). Il m'a signalé des travaux sur l'architecture domestique comme ceux de Gwendolyn Wright sur la maison américaine ${ }^{15}$ ou de Donatella Calabi sur I'Angleterre ${ }^{16}$. Pour étudier l'habitation en France, je croyais, naïvement, qu'il me suffirait de remonter une trentaine d'années en arrière afin de comprendre comment certaines dispositions des logements contemporains s'étaient imposées. J'ai ainsi engagé une enquête avec l'aide d'une de mes étudiantes, Anne Debarre. L'investigation nous a conduites, de fil en aiguille, à remonter très en amont, jusqu'au début du XVII siècle, au moment où Pierre Le Muet publie Manière de bien bastir pour toutes sortes de personnes en $1623^{17}$. La publication des deux tomes d'Architectures de la vie privée est le résultat de ce travail couvrant, d'une part, les XVII et XVIII e siècles (ELEB-VIDAL, avec DeBARRE-BLANCHARD, 1989), d'autre part, 
le XIXe et le début du XXe (ELEB, avec DEBARRE, 1995). Les sources incluaient l'étude des traités et recueils de la période ainsi que les ouvrages d'histoire et la littérature, l'analyse des plans et des dénominations, mis en regard des représentations en tout genre : tableaux, gravures, dessins puis photographies...

- Estelle Thibault. Parmi les différentes sources mobilisées, vous avez donc fait une place non négligeable aux documents visuels. Qu'est-ce que leur analyse apportait de spécifique par rapport aux travaux et documents écrits qui abordaient alors l'histoire des mentalités?

- Monique Eleb. Les groupes de chercheurs auxquels j'ai appartenu, notamment autour du laboratoire de Sciences humaines cliniques de l'université Paris VII, dirigé par Claude Revault d'Allonnes, refusaient la division des sciences humaines en spécialités. Nous estimions que toutes les méthodes, tous les concepts permettant de comprendre un phénomène ou un terrain pouvaient être mobilisés. C'est un aspect fondamental de mon parcours. Or l'habitat touche à des dimensions à la fois sociale, psychologique, urbanistique, politique, historique... Dans ce contexte, j'ai tenté quelque chose que je n'aurais jamais osé tenter dans ma discipline d'origine, à savoir, analyser

1. Pieter de Hooch, La Chambre, $1658 / 1660$, Washington, National Gallery of Art.

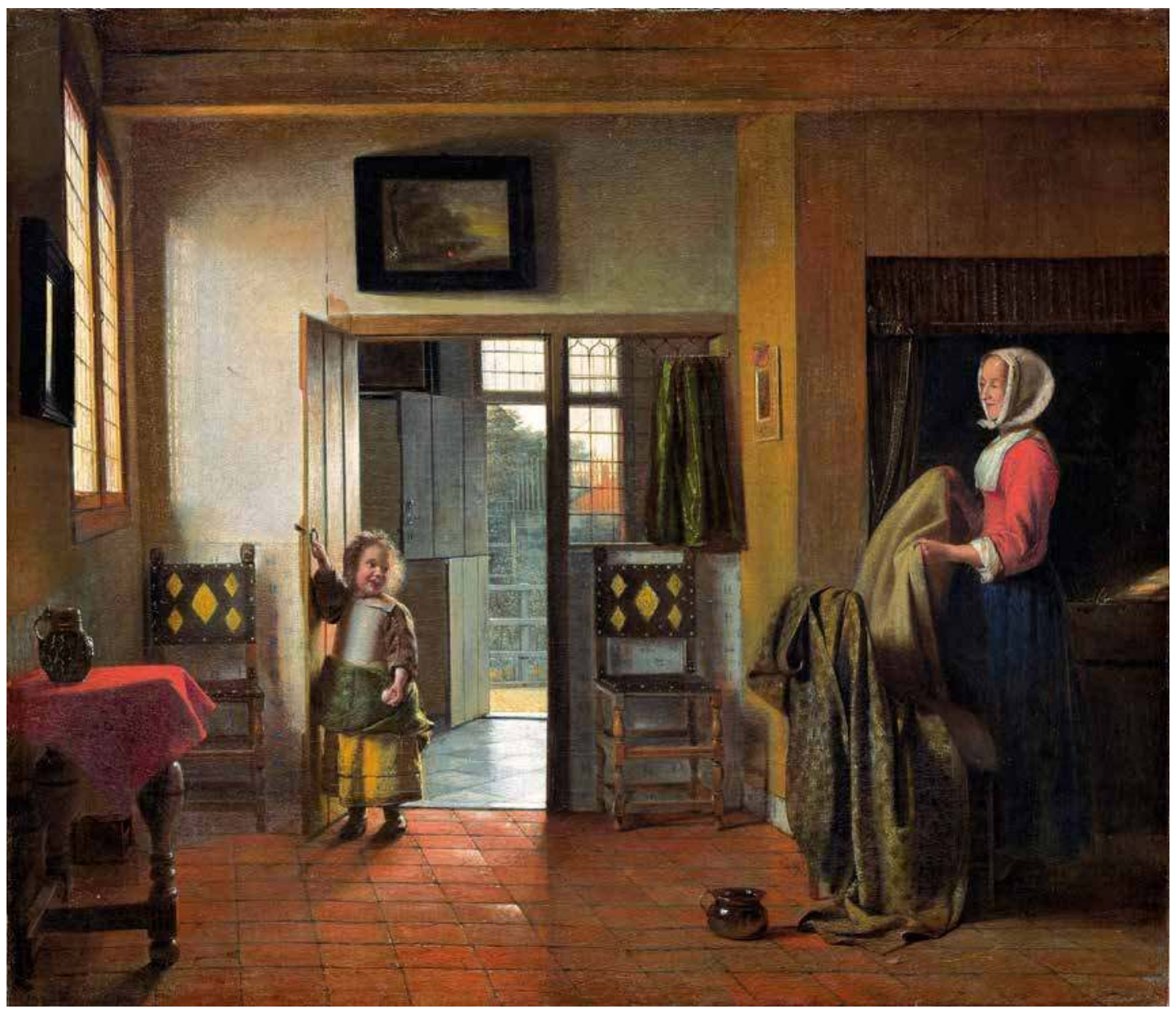


les plans et les images. Alors que je travaillais au premier tome d'Architectures de la vie privée, j'ai eu l'opportunité de parcourir de nombreux fonds iconographiques à la recherche d'illustrations, de plans, de tableaux. L'étude de ces documents est vite devenue essentielle. L'analyse des plans d'architecture nécessitait un apprentissage, mais s'est révélée assez facile dès lors que j'avais compris l'évolution des dénominations sur les plans et que j'ai considéré que ces derniers organisaient le mouvement du corps et permettaient de comprendre la situation et le statut des personnes. Je m'intéressais à la manière dont les architectes, parfois de façon inconsciente, traduisaient dans l'espace un système sociologique, les règles de la vie quotidienne dans un pays donné, les rapports de genre entre les hommes et les femmes, les relations entre parents et enfants, maîtres et domestiques. Je me suis mise à faire de la généalogie, c'est-à-dire à tenter de comprendre comment on passait d'un comportement à l'autre, d'un plan à un autre, d'une dénomination des pièces à d'autres.

La lecture de Pierre Francastel, qui considérait les œuvres d'art comme des objets de civilisation ${ }^{18}$, m'a complètement délivrée de mes craintes d'illégitimité en la matière. Le plan aussi était un fait de civilisation, témoignant des interrelations entre les personnes, de ce qu'elles veulent montrer ou occulter. Les plans dessinés par les architectes changent quand les manières de vivre évoluent. Ainsi, dans des recueils ou des traités d'architecture publiés à vingt ans d'intervalle, les plans des mêmes hôtels pouvaient avoir été corrigés, ce que personne ne semblait avoir remarqué. Cela confirmait l'hypothèse selon laquelle l'habitat incarne et représente les rapports interindividuels, interhumains. Henri Lefebvre l'observait également à propos des plans d'urbanisme.

Aux côtés des plans, j'ai aussi beaucoup utilisé les tableaux, par exemple ceux des Hollandais du XVI ${ }^{e}$ siècle, qui, parmi les premiers, représentaient des intérieurs (fig. 1). Je me suis intéressée aux symboles déployés par le peintre pour figurer certains sentiments comme la fidélité ou pour introduire des connotations sexuelles. Dans ces scènes d'intérieur, les objets, les postures, I'habillement, tout était objet de civilisation. Ces tableaux décrivaient aussi l'organisation du plan, puisqu'ils mettaient souvent en scène le rapport d'une pièce à l'autre (fig. 2).

Le premier tome d'Architectures de la vie privée comprend un complément qui expose la méthode. Elle a consisté à croiser les sources, à mettre en relation les plans, les tableaux, les textes de l'époque en portant une attention particulière aux dénominations des espaces et à leur évolution. Cette méthode permettait d'identifier les moments de rupture.

J'ai pris conscience que je travaillais sur les mœurs et sur l'histoire des mentalités, à I'instar de Philippe Ariès ou de Michelle Perrot ${ }^{19}$, mais à partir de l'architecture. À cette époque-là, Lion Murard et Patrick Zylberman avaient effectué une recherche sur l'intimité en considérant que la notion émergeait au XVIIII siècle ${ }^{20}$. Or les plans et les images, gravures ou peintures, indiquaient une origine bien antérieure dans I'habitation aristocratique. Le partage de l'habitat en zones privées et zones de représentation semblait s'opérer en France dans la première moitié du XVIII siècle, avant de se diffuser lentement. L'analyse des plans de maisons de ville montrait la présence de pièces de retraite, cabinets, oratoires, garde-robes. La volonté d'être seul, de faire retraite, pouvait être corrélée à des questions religieuses : la Contre-Réforme, en prohibant la nudité ou la trop grande promiscuité, impose des contraintes morales à la vie domestique, ce qui fait évoluer les demandes formulées aux architectes. Une autre rupture fondamentale de l'histoire de l'architecture domestique française intervient avec l'apparition des couloirs qui permettent de distribuer les pièces autrement que par un système d'enfilade, rendant les pièces indépendantes les unes des autres en doublant les flux. Ainsi JacquesFrançois Blondel désigne-t-il le plan du palais Bourbon (1722) comme un exemple remarquable en termes de « commodité » (fig. 3).

2. Félix Vallotton, Intérieur avec une femme en rouge de dos, 1903, Zurich, Kunsthaus. 


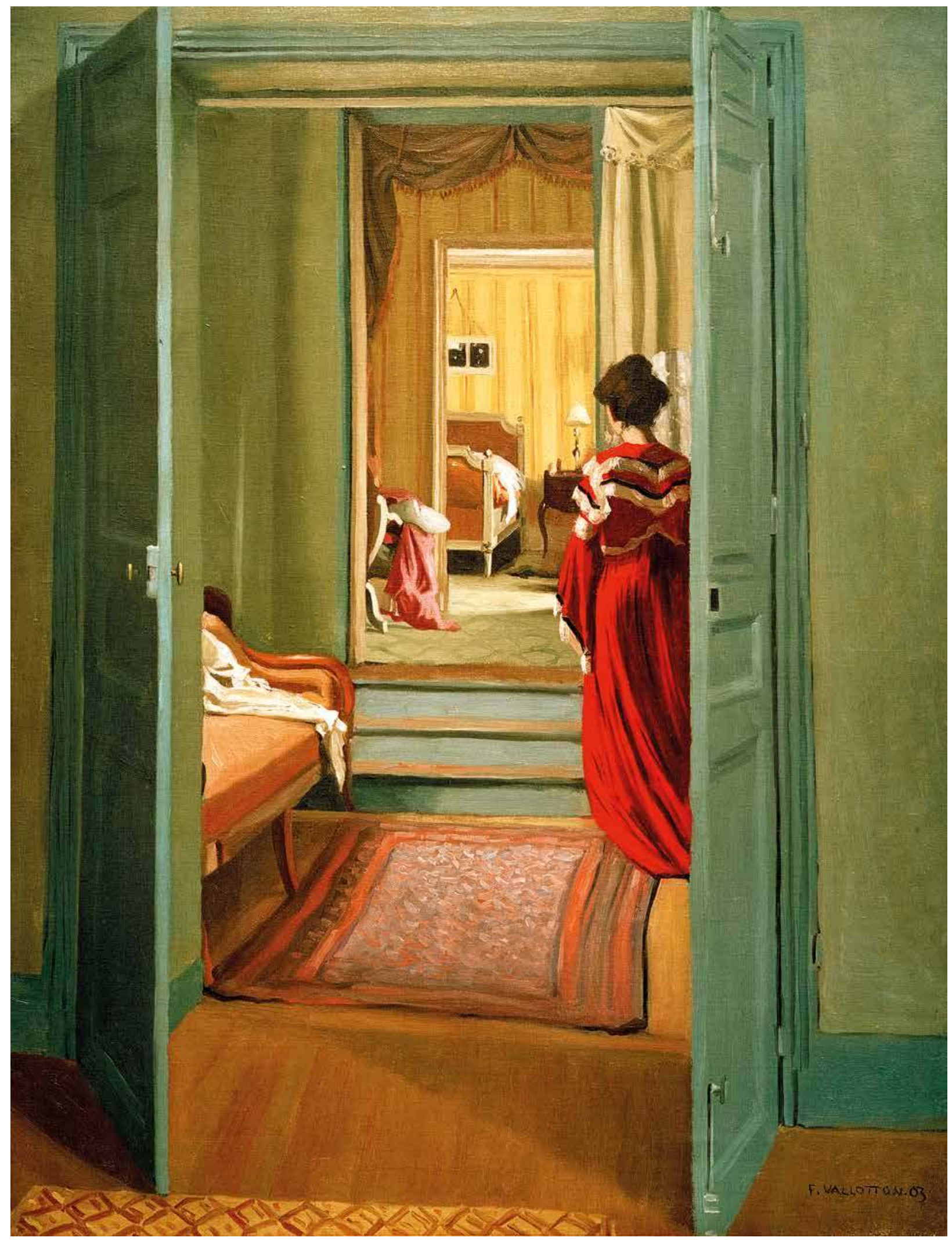




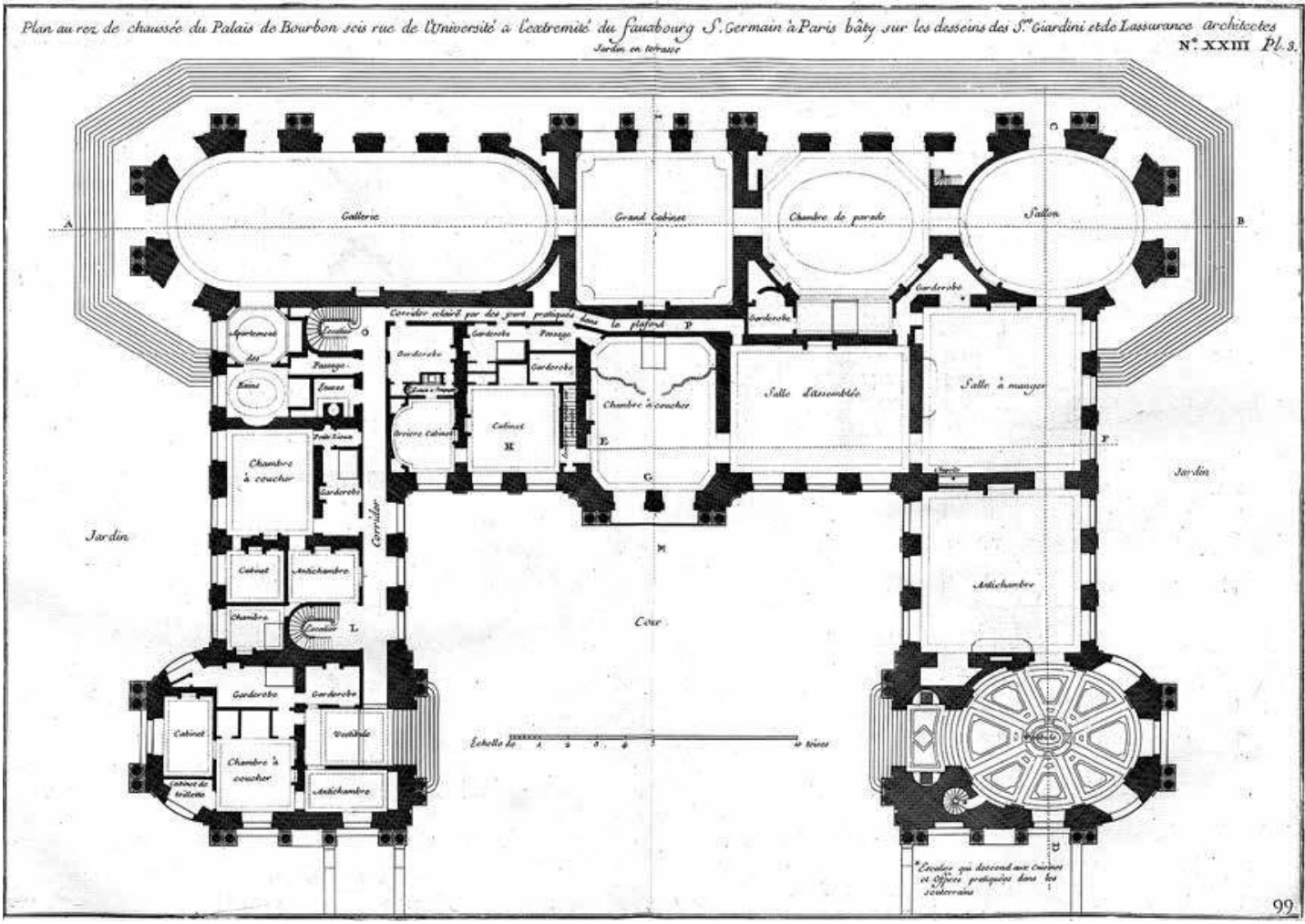

3. «Plan du rez-de-chaussée du Palais Bourbon", dans Jacques-François Blondel, Architecture françoise..., 1752-1756, Paris, Charles-Antoine Jombert, t. I, chap. XXIII, pl. 3.
Curieusement, j'ai lu assez tard La Société de cour de Norbert Elias ${ }^{21}$, qui décrit avec une certaine acuité les relations entre les mœurs et les dispositifs spatiaux à partir d'articles issus de I'Encyclopédie ou d'autres textes littéraires. II met par exemple en évidence le fait que les époux vivent dans des appartements totalement séparés, de part et d'autre de l'hôtel, et n'ont que peu de contacts ; ils évoluent dans des cercles sociaux distincts et communiquent par l'intermédiaire des domestiques ${ }^{22}$. La conception du mariage se trouve matérialisée par l'architecture, ce que vient confirmer l'étude des plans.

\section{- Estelle Thibault. Pourriez-vous préciser comment vous utilisez la notion de dispositif ?}

- Monique Eleb. La notion de dispositif au sens où je l'emploie a moins à voir avec Michel Foucault, qui insiste sur les rapports de pouvoir, qu'avec le dispositif de la cure psychanalytique freudienne ${ }^{23}$. Ici le dispositif est compris comme l'organisation d'éléments spatiaux assemblés pour produire un effet particulier. Ainsi, dans la cure, l'emplacement des meubles détermine celle des corps ; les regards ne doivent pas se croiser, la position des objets ou des tableaux constitue une mise en scène spatiale qui est censée produire des effets particuliers. De même, les agencements des espaces, des murs, des portes et des fenêtres dans les appartements privés organisent les déplacements et les relations interindividuelles. Cette notion de dispositif a été très utile à un grand nombre d'étudiants et de doctorants pour analyser I'habitat mais aussi, bien au-delà, l'architecture en général, celle des musées par exemple. 
- Estelle Thibault. Vous qualifiez souvent votre démarche de recherche en généalogie. Pourriez-vous préciser en quoi vous la distinguez d'une approche historique ? S'agit-il de procéder à rebours?

- Monique Eleb. Il s'agit moins de procéder à rebours que d'étudier les transformations sur un temps relativement court et de porter son attention sur les processus d'engendrement. Mon objectif premier était de comprendre comment on passe d'un dispositif à un autre et les raisons des changements. Les modifications dans la manière de distribuer l'habitation et de désigner les pièces sont révélatrices de transformations profondes des mentalités et des modes de vie. Dans le premier tome d'Architectures de la vie privée figure l'exemple de l'hôtel de Béthune, publié par Jean Mariette en 1727 puis par Jacques François Blondel vingt-cinq ans plus tard, avec de nombreuses modifications dans les dénominations des pièces (fig. 4 et 5). Cela traduit des évolutions dans les pratiques dont les architectes tenaient compte, en spécialisant de plus en plus les pièces (chambre à coucher, salle d'assemblée, cabinet, arrière-cabinet...) et en les autonomisant, alors qu'auparavant elles se « commandaient » les unes les autres. Par cette approche généalogique, j'ai osé faire quelque chose qui se faisait peu en histoire de l'architecture, à savoir travailler sur le temps long. Les historiens se centraient souvent sur des segments temporels plus brefs.

- Estelle Thibault. Les périodes anciennes vous ont conduite à recourir à d'autres types de sources et à déployer une certaine inventivité en termes de méthode. Réciproquement, les investigations sur ces périodes ont-elles stimulé des questionnements féconds pour l'analyse du logement contemporain?

- Monique Eleb. Plus largement, mon travail s'est fondé sur l'appropriation de concepts provenant d'autres domaines que l'architecture mais qui se sont révélés très féconds pour analyser celle-ci. Peu à peu se sont mis en place un vocabulaire et une méthode d'analyse qui permettent de lire un plan, de le
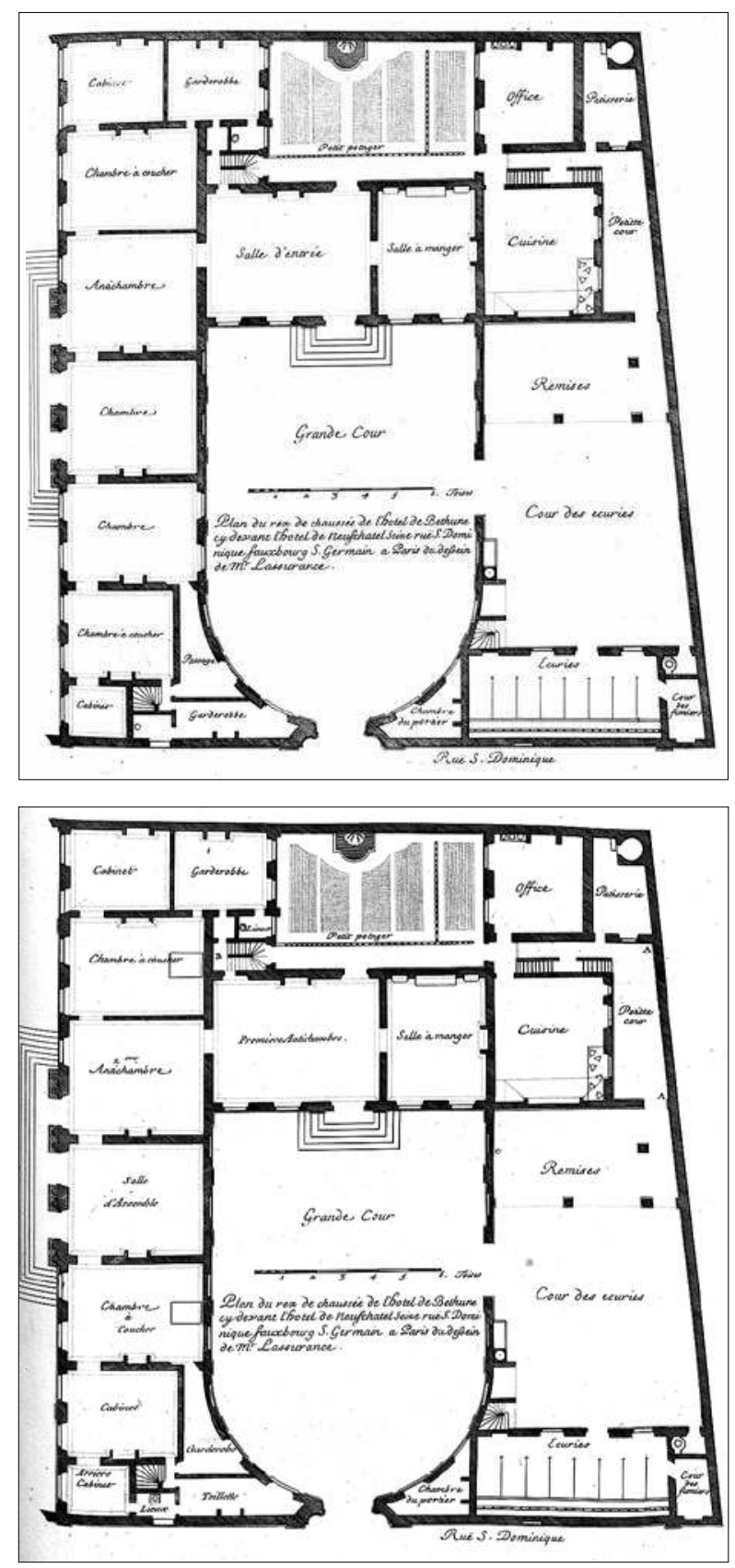

4. "Détail du plan du rez-de-chaussée de l'hôtel de Béthune ", dans Jean Mariette, Architecture françoise..., Paris, Jean Mariette, 1727.

5. " Détail du plan du rez-de-chaussée de l'hôtel de Béthune ", dans Jacques-François Blondel, Architecture françoise..., Paris, Charles-Antoine Jombert, 1752-1756, n XVII, pl. 1. 

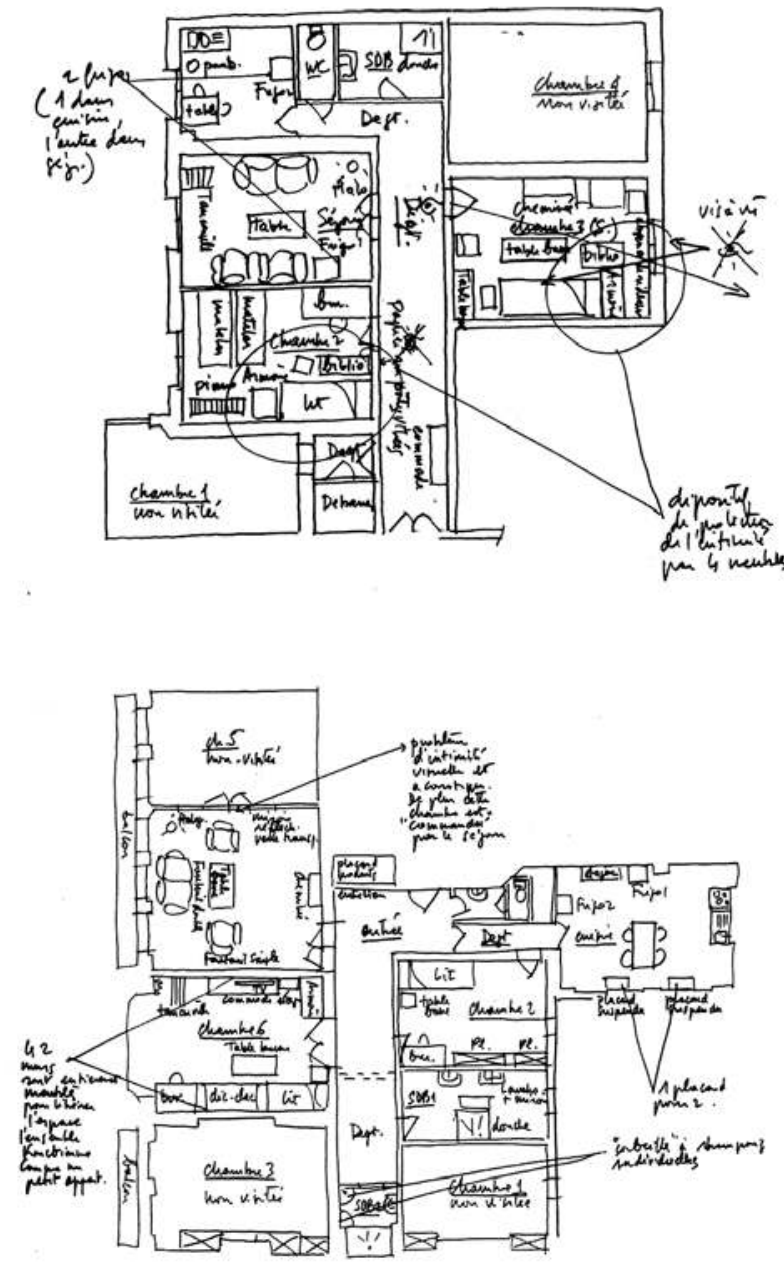

6. « Deux relevés de plans habités, appartements pour colocataires étudiants du boulevard de Port-Royal à Paris », dans ELEB, BENDIMÉRAD, 2018, p. 25. dater, de comprendre quel type de famille ou quelle population peut y habiter, à partir des relations qu'il induit entre les personnes. Pour les périodes anciennes, il est nécessaire de croiser différents types de sources en effet : les plans montrent la partition de l'espace et la distribution, la taille et la position relative des pièces. Les dénominations des lieux, inscrits sur les plans, révèlent certaines pratiques. L'analyse des discours contenus dans les traités, revues, dictionnaires et autres ouvrages, renseigne sur les doctrines et sur les pratiques. Les tableaux et gravures qui mettent en scène la vie quotidienne fournissent des informations qu'il faut décoder prudemment, en prenant garde aux anachronismes. Les éléments les plus pertinents sont souvent à chercher à l'arrière-plan, qui montre les dispositions spatiales, ou dans des détails indiquant les façons de vivre et d'habiter. C'est la mise en série de ces informations, croisées avec d'autres sources, qui permet de comprendre l'organisation des espaces.

Pour travailler sur l'architecture contemporaine, il est souvent nécessaire de créer les sources. L'étude des plans, la visite, les relevés de plans habités (fig. 6), mais aussi les entretiens avec les concepteurs ou les habitants s'avèrent précieux, même si l'étude de la parole des concepteurs, comme celle des usagers, doit être appréhendée avec précaution. L'apport essentiel du détour par les périodes anciennes a été de travailler sur les idéologies et les représentations, ce à quoi mes études de psychologie et de sociologie m'avaient bien préparée. J'ai également mobi-

lisé mes outils de psychologue pour étudier les périodes plus anciennes. Mes connaissances sur la perception, sur le mouvement et sur les représentations mentales m'ont été très utiles pour analyser les représentations visuelles.

\section{- Estelle Thibault. Cette approche sur le long terme n'est-elle pas indispensable pour aborder} I'innovation?

- Monique Eleb. Travailler sur une période étroite entraîne en effet un biais important, qui consiste à croire nouveaux des dispositifs qui existaient déjà de longue date, parfois cinquante ou cent cinquante ans auparavant... Le débat architectural actuel met l'accent sur les notions de réversibilité ou de flexibilité, notamment. Or au XIXe siècle, les propriétaires demandaient déjà aux architectes de concevoir des appartements que l'on pourrait agrandir ou réduire au moment de la vente ou du changement de locataire, en réunissant diverses pièces par 
un système de portes, par exemple. Ces réflexions étaient loin d'être nouvelles. Les véritables changements se préparent sur la longue durée, et sont acceptés et diffusés lorsque la société est prête à les accueillir. Je peux illustrer cette idée autrement. J'ai effectué en 1987 I'analyse de la quatorzième édition du Programme d'architecture nouvelle (PAN 14) ${ }^{24}$ intitulée « Le logement en questions : type, contexte, modification ", dont un thème récurrent était celui de la souplesse d'utilisation grâce à des espaces techniques intégrés (ELEB-VIDAL, CHÂTELET, MANDOUL, 1988 ; fig. 7). Sur le moment, les propositions ont certes intéressé les théoriciens et les chercheurs, en France comme à l'étranger, mais n'ont pas atteint le grand public. Quant aux maîtres d'ouvrage et aux promoteurs, ils n'étaient pas enclins à proposer des changements aussi radicaux. Aujourd'hui, les revues de décoration ne parlent que de réversibilité, de flexibilité : déplacer les murs, ouvrir, modifier... Les tentatives expérimentales qui étaient marginales il y a trente ans se retrouvent également de façon récurrente dans la production architecturale récente (ELEB, SIMON 2013 ; ELEB, BENDIMERAD, 2018).

Cela pose la question de l'innovation, abordée par Gilbert Simondon dans les cours auxquels j'ai eu la chance d'assister, puisqu'il enseignait au département de psychologie de la Sorbonne dans les années 1960. Cette question est cruciale. Que désigne-t-on comme innovant, que valorise-t-on comme idéal et comment cela est-il reçu par la population ? Dans quelles circonstances apparaît une innovation ? Qui la motive ? Travailler avec des maîtres d'ouvrage et des architectes m'a permis de comprendre qu'aujourd'hui les habitants commencent à être plus moteurs dans la demande de changement. Depuis dix ou quinze ans, certains promoteurs ou maîtres d'ouvrage publics sont amenés à innover pour s'adapter à cette demande, car ils se sont rendu compte que ce qu'ils proposaient était souvent de qualité médiocre, ou moins bon qu'avant. La demande d'innovation peut également émaner de quelques maîtres d'ouvrage "d'art et d'essai », peu nombreux, et j'ai eu beaucoup de plaisir à travailler sur la conception de logements avec certains d'entre eux, ainsi qu'avec les équipes d'architectes et les autres intervenants.

- Estelle Thibault. La qualité des logements a-t-elle régressé ces dernières décennies ? L'expérience du confinement, enfin, a-t-elle modifié la demande?

7. Catherine Lauvergeat et Thierry Nabères, "Les cohabitants, scénario du projet "Un + Un ou comment réussir sa cohabitation" ", dans ElEB-VIDAL, CHÂTELET, MANDOUL, 1988, p. 49.

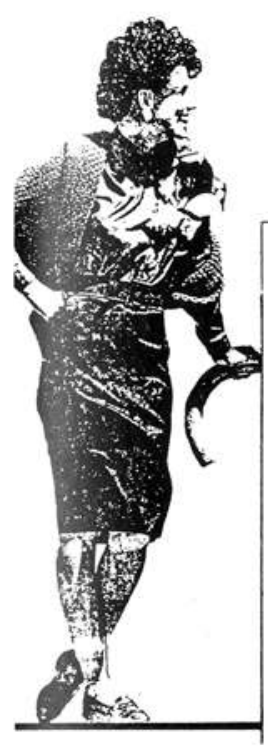

Les cohabitants
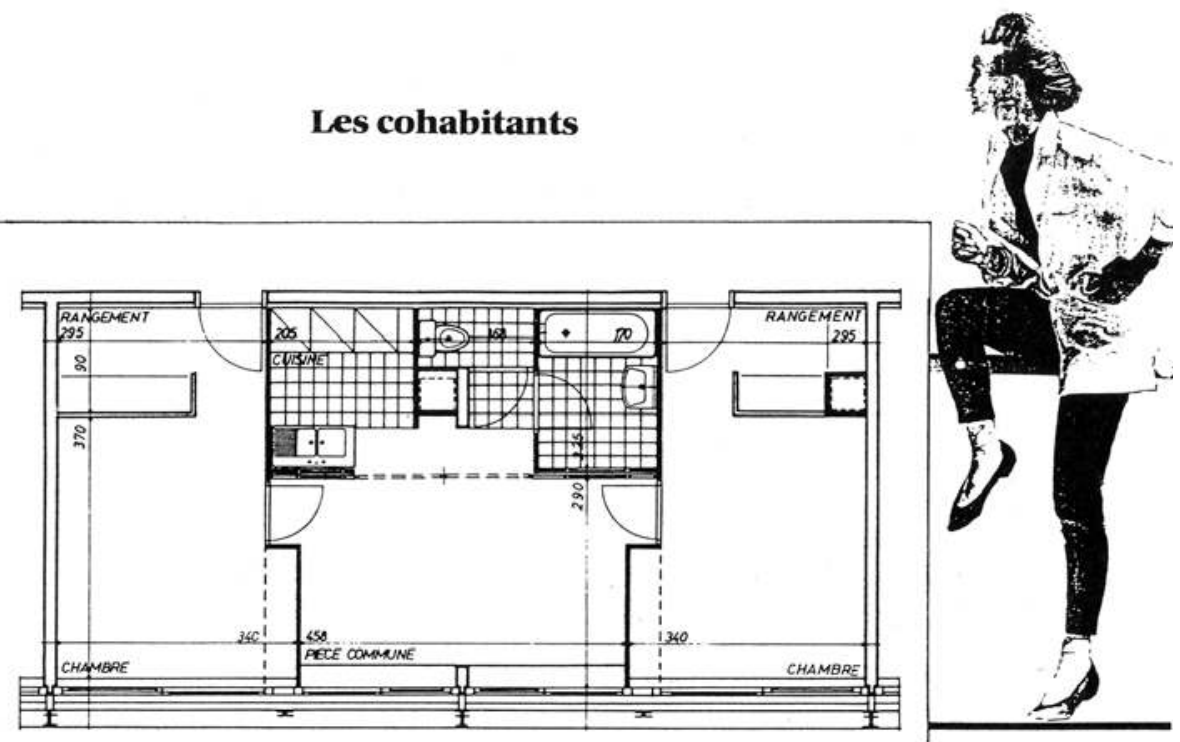
- Monique Eleb. Tout récemment, le confinement a entraîné une prise de conscience de toute part, d'abord du manque de rapport à un extérieur privatisé, comme une terrasse, une loggia, etc. Cela peut être lié au fait que les hommes, plus présents que d'ordinaire à la maison, ont enfin éprouvé le manque de qualité des logements et en ont souffert, et au fait que les femmes ont vu s'accentuer la pression concernant le travail ménager. Cela pourrait accélérer le processus d'évolution. Depuis quelques années le recul de la qualité des logements est important à plusieurs titres. Certaines pièces comme la cuisine sont en train de disparaître, alors que depuis trente ans mes enquêtes de terrain $\mathrm{m}^{\prime}$ indiquent que beaucoup préfèrent avoir des cuisines séparées. Pour des questions de coût, on propose une ligne d'électroménager au fond d'un séjour, ce qui permet en réalité de réduire la superficie. Dans l'un des derniers bâtiments que j'ai étudiés, 90\% des cuisines ouvertes avaient été refermées au bout d'un an car la grande majorité des habitants ne les appréciaient pas. L'âge et la classe sociale des habitants jouent un rôle autant dans l'acceptation que dans le refus de ce type d'aménagement. Il faudrait en fait prévoir des cuisines ouvertes que l'on peut fermer. De plus, à Paris, les surfaces des logements neufs ont baissé de 5 mètres carrés minimum au cours de la dernière décennie. Les évolutions sont sans doute à penser sur diverses échelles de temps.

- Estelle Thibault. Vous avez également contribué à former les architectes sur ces questions. Vos travaux, mais aussi vos enseignements dans les écoles d'architecture, ont conduit vos étudiants et vos collègues à prendre conscience du fait qu'ils intériorisaient des normes et les ont invités à se réapproprier le champ de l'architecture domestique.

- Monique Eleb. L'enseignement a été très important dans mon parcours, avec de nombreuses batailles dont la plus épique fut celle du doctorat en architecture. Très vite, parallèlement à mes recherches, j'ai souhaité enseigner avec des architectes. Je constatais qu'il existait une architecture savante qu'on avait oubliée. Plus personne ne lisait les traités, il n'y avait plus d'enseignement de théorie de l'architecture domestique alors qu'au XIXe siècle certains périodiques, comme la Revue générale de l'architecture et des travaux publics de César Daly, d'un niveau extraordinaire, étaient le support d'un apprentissage très fin de l'architecture de l'habitation. En amont et depuis Pierre Le Muet au XVII siècle, les traités et recueils constituaient un important fonds théorique utilisé par les concepteurs. Je souhaitais que ces savoirs soient diffusés et actualisés. C'est pourquoi je me suis rapprochée d'architectes qui travaillaient sur l'habitat dans les différentes écoles d'architecture. En 1984, avec Patrick Céleste, nous avons créé le premier CEEA (certificat d'études approfondies en architecture) "Architecture domestique », avec Jean Castex, Claude Prélorenzo, et d'autres. Ces architectes avaient en commun d'être sensibilisés aux sciences humaines, soit parce qu'ils côtoyaient des sociologues et psychologues dans leur entourage proche, soit parce qu'ils s'étaient eux-mêmes formés, comme Christian Moley qui avait soutenu une thèse en ethnologie. Ils étaient également capables de dépasser les questions de genre qui existent de longue date entre architecture militaire et civile, l'une monumentale, adressée aux hommes et constitutive de l'espace public et l'autre domestique, tournée vers l'intérieur et pensée comme étant destinée aux femmes. Cette équipe a posé, avec d'autres, les bases de la création du DEA (diplôme d'études approfondies) inter-écoles d'architecture « le projet architectural et urbain, théories et dispositifs », en 1991. Ce DEA fut l'objet d'un véritable combat car le ministère de la Culture, qui est la tutelle des écoles d'architecture, n'y était pas favorable. L'architecture domestique était l'un des trois axes, aux côtés de l'étude de l'architecture urbaine et des villes orientales. Bien avant la mise en œuvre en septembre 2005 de la réforme qui instaura le doctorat dans les écoles d'architecture, cette formation qui promouvait l'interdisciplinarité formait déjà de nombreux docteurs qui enseignent aujourd'hui en France et à l'étranger. 
Au sein de ce DEA, j'étais chargée non seulement d'un cours sur l'histoire et l'analyse de I'habitat en France et sur la conception aujourd'hui, mais aussi des enseignements méthodologiques. Ils étaient fondamentaux puisque la plupart de nos étudiants, architectes de formation, étaient plus habitués à résoudre des problèmes qu'à adopter des raisonnements hypothéticodéductifs. Il s'agissait de les transformer en chercheurs... Ce fut une formidable aventure mais elle nous a beaucoup coûté.

- Estelle Thibault. Pourrions-nous également aborder les différentes formes d'engagement qui ont motivé vos travaux? Le choix même du sujet, l'habitation, peut être commenté de ce point de vue, ainsi que le fait que vous avez souvent mis l'accent sur les rapports de genre, en consacrant un chapitre de chacun de vos livres à la place des femmes.

Monique Eleb. Mon premier engagement fut de travailler à ce que l'enseignement de l'architecture soit digne et reconnu comme aussi qualitatif que des enseignements de haut niveau à I'université. L'Allemagne avait mis en place un doctorat en architecture à la fin du XIXe siècle et, à la fin des années 1980, nous n'en avions toujours pas en France. Être à l'origine de la fondation de l'École de Paris-Malaquais en 2000, en prenant une part active aux instances, y défendre l'importance d'une recherche interdisciplinaire face à des collègues architectes praticiens, tout cela demandait un certain engagement, de même que créer une équipe de recherche, travailler à son affiliation au CNRS, participer à la mise en place d'un doctorat.

Si la reconnaissance par le ministère de la Culture n'a pas été à la hauteur des efforts, le fait que mes idées aient pu contribuer à améliorer le logement pour le grand public a été une véritable satisfaction car certains des concepts que j'ai essayé de promouvoir depuis longtemps, comme la flexibilité ou la réversibilité du logement, l'adaptabilité aux usages, sont en train de se banaliser. Choisir l'habitation comme sujet était un choix militant car si le logement était le terrain le plus négligé par la recherche, il était le plus important pour les gens. Combien de fois les habitants, sur le terrain, ont-ils déploré que l'effort architectural ait été porté sur la façade plutôt que sur les qualités de l'intérieur ? Je pense par exemple à une visite faite avec les étudiants dans une opération de réhabilitation d'un immeuble (à Paris, dans le XIII a arrondissement) réalisée par Christian de Portzamparc à la fin des années 1990. Il y a une incompréhension mutuelle entre les architectes et les habitants car le soin apporté à la façade, au hall, à la manière d'entrer, améliore notablement la qualité, mais les habitants sont plus sensibles à l'état de leur salle de bains. Du reste, ces incompréhensions entre architectes et habitants ne sont pas nouvelles. Paul-Henry Chombart de Lauwe le constate déjà dans Famille et habitation, en interrogeant divers architectes ${ }^{25}$. Lorsque Charlotte Perriand se demande pourquoi les Français refusent de vivre comme les Japonais, cela démontre sa complète incompréhension du fait que l'habitat est géographiquement et culturellement déterminé.

Enquêter sur la place des femmes a également été fondamental. Le soin de l'intérieur de la maison leur est associé, c'est un fait de civilisation. Il n'y a pas de femmes architectes avant la fin du XIX ${ }^{\mathrm{e}}$ siècle. Pour autant, les premières d'entre elles, comme Julia Morgan en Californie, concevaient-elles des espaces différents de ceux imaginés par les hommes? En amont, l'étude des pièces dédiées aux femmes est essentielle pour comprendre l'évolution de l'habitation. Aux XVle et XVII siècles, les oratoires, qui étaient initialement des lieux de prière féminins, se transforment en cabinets où les femmes aristocrates lisent et écrivent, ce qui traduit une véritable transformation de la place de celles-ci. Le XIXe siècle marque un certain recul du statut des femmes, lisible notamment dans l'Histoire d'une maison $d^{\prime}$ Eugène Viollet-le-Duc ${ }^{26}$. II place la chambre du « maître de maison » du côté de la plus belle orientation, quand auparavant c'était le privilège de la femme de bénéficier de la meilleure vue. Il donne un petit balcon à celle-ci et une grande loge à celui-là. II n'y a plus 
de boudoir, mais une salle de billard et un bel espace où ces messieurs pourront fumer. Aucun de mes collègues masculins, qui trouvaient formidable cette Histoire d'une maison, ne l'avait remarqué.

Mais parler des hommes et des femmes a peu à voir avec du militantisme. II me semblait normal de parler de leurs statuts et leurs rôles étant donné ma formation. J'ai fait ma thèse en sociologie de la connaissance et des idéologies, ce qui impliquait d'analyser les représentations, les idées. Le quotidien et le banal sont les choses les plus difficiles à comprendre et à transformer tant elles paraissent naturelles. Analyser les maisons permet de retracer I'histoire des statuts respectifs des femmes et des hommes. Où se lavaient ces derniers ? Les traités de savoir-vivre, excellents révélateurs des idéologies, sont muets ou presque sur cette question, car les lieux pour se laver étaient dédiés aux femmes, qui tout au plus y prêtaient un petit coin à leur mari autour de 1900 (ELEB, avec DEBARRE, 1995, p. 225). Des espaces dédiés à la propreté des hommes apparaissent dans I'habitation ouvrière, quand les populations rurales arrivent en ville et qu'il devient nécessaire de leur inculquer des habitudes de propreté. Les indications révélées par les traités de savoir-vivre sont confirmées par les plans, qui reflètent une idéologie non verbale. L'examen des documents graphiques permet de décoder des symboles, des représentations et des systèmes de relations hommes-femmes, mais aussi parents-enfants ou maîtres-domestiques. Peut-être parce que j'avais moi-même eu des domestiques quand j'étais enfant, je ne me suis pas aperçue immédiatement à quel point ceux-ci étaient fondamentaux dans la vie des maîtres, ce que révèle pourtant la littérature, le théâtre tout particulièrement. Je l'ai compris pleinement en abordant la période où est inventé le double flux de circulation, qui permet aux domestiques de circuler par des chemins indépendants de celui des maîtres. L'invention du couloir est une révolution qui permet de ne pas se croiser et que le service ait lieu dans les coulisses de la maison. Le bâtiment d'Auguste Perret, au 26 bis, rue Franklin à Paris (XVle arrondissement),

8. Auguste Perret, "Plan du 26 bis, rue Franklin, Paris ", L'Architecture d'aujourd'hui, n 7, octobre 1932, p. 19. est exemplaire à ce titre (fig. 8). Les pièces principales, situées en enfilade sur la façade, forment une scène avec, à I'arrière, les coulisses. C'est avec cette méthode, ce regard

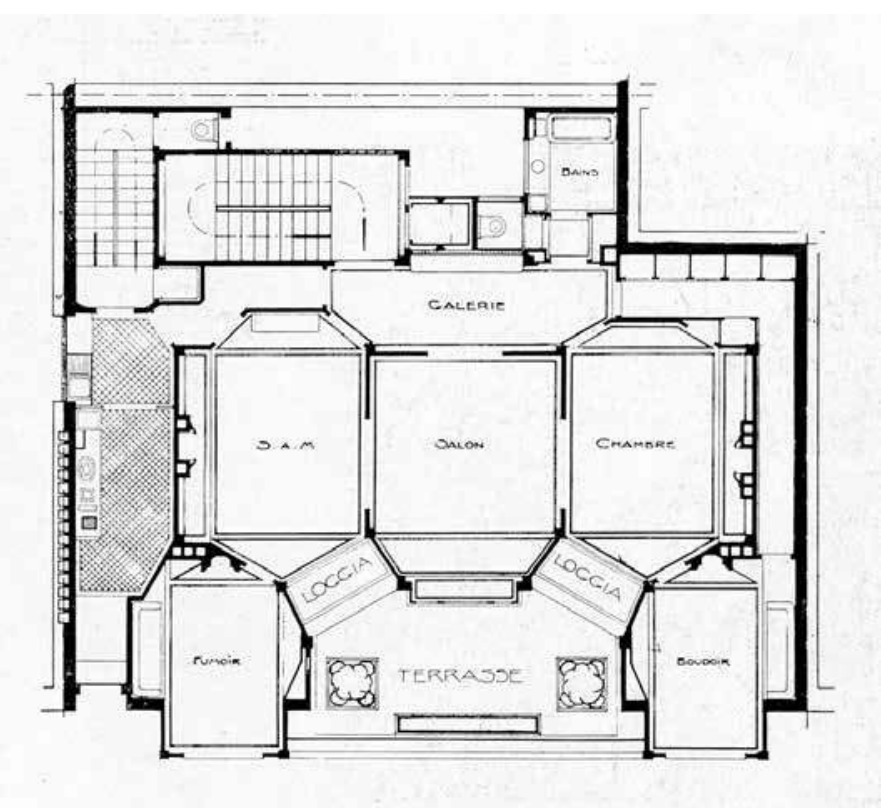




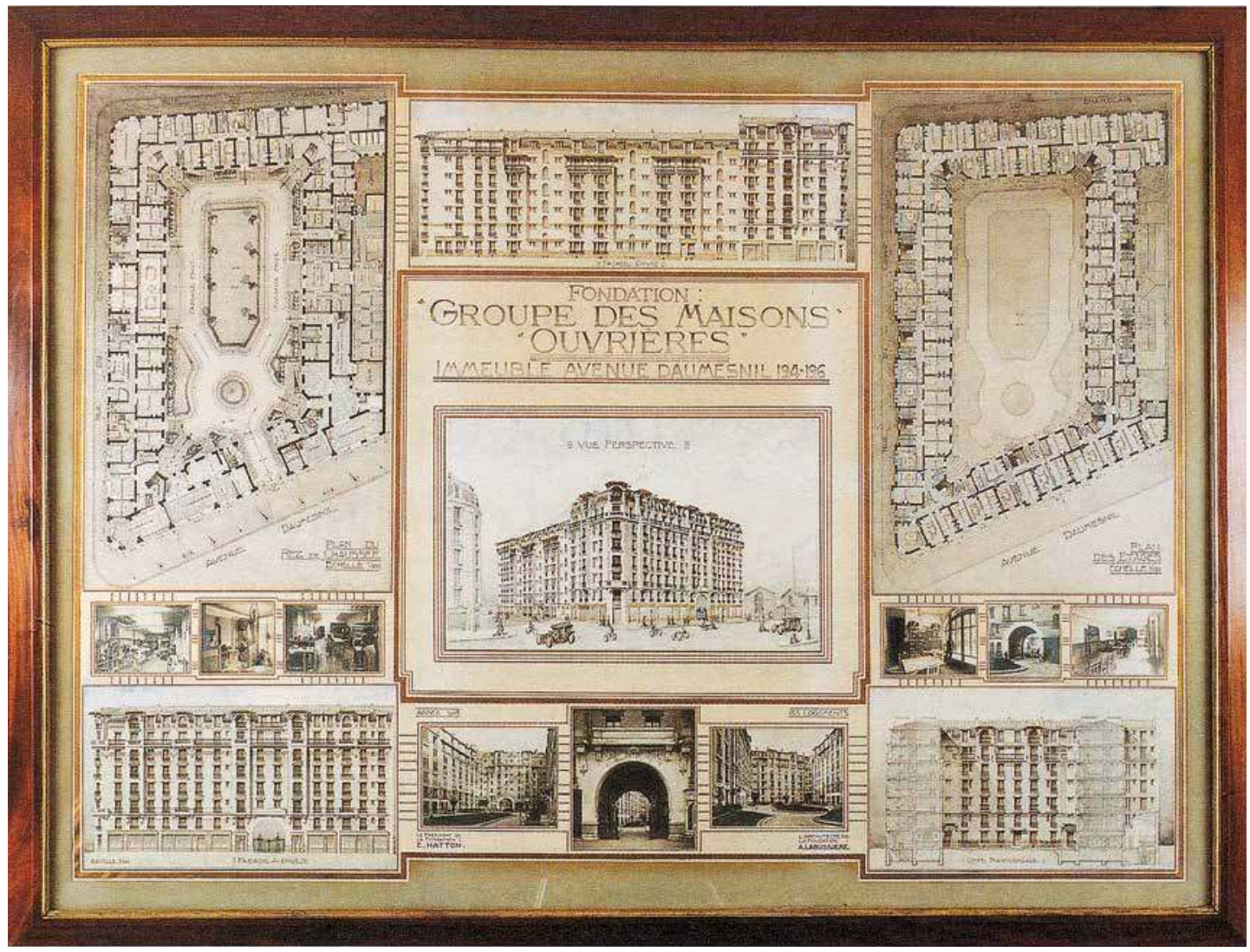

sur certaines organisations, que j'ai ainsi pu mettre en avant des aspects qui jusqu'alors n'avaient été que peu commentés. Ce que j'espère, c'est que cela s'est transmis.

9. «Tableau de présentation du projet de Groupe des maisons ouvrières de l'avenue Daumesnil ", Paris, dans ELEB, 1994, p. 75.

- Estelle Thibault. Vos travaux sont caractérisés non seulement par le fait d'étudier simultanément diverses périodes, mais aussi de regarder parallèlement les habitations de différentes classes sociales. Vous vous intéressez également à la manière dont la conception et les programmes de logements, élaborés conjointement par les commanditaires et les architectes, constituent des tentatives de réformer les comportements.

- Monique Eleb. Il n'a pas été simple de travailler sur les périodes où la noblesse dicte les conduites, à une époque où la recherche en architecture s'intéressait principalement au monde ouvrier. Comme j'avais fait une thèse sur l'habitat social, certains me reprochaient d'avoir trahi. Mais pour appréhender pleinement l'architecture de la classe ouvrière, il m'est vite apparu qu'il fallait comprendre comment les différents systèmes s'articulent, comprendre que le logement ouvrier avait été conçu comme une réduction de l'habitation bourgeoise. Dans le livre L'Apprentissage du chez-soi, qui portait sur le groupe des maisons ouvrières de l'avenue Daumesnil à Paris (XII' arrondissement), j'ai principalement mis en évidence le regard de la bourgeoisie sur la classe ouvrière au début du XXe siècle (ELEB, 1994 ; fig. 9). II s'agissait d'une remise au pas, d'apprendre à habiter et de conformer les comportements. Pour comprendre les dispositifs proposés il était nécessaire, au préalable, d'avoir une idée 
claire des modes de vie et d'habitation des nantis. Cela permettait de saisir ce qui avait été supprimé ou réduit dans les projets d'habitation ouvrière.

Les oppositions que certains de mes travaux ont suscitées me confortent dans l'idée que mon approche était légitime et que dépasser les limites méthodologiques traditionnelles des disciplines des sciences humaines, en faisant feu de tout bois en termes de méthode, permettait de mieux travailler sur la réalité et sur l'architecture, qui est un objet profondément interdisciplinaire et inscrit dans le temps long.

- Estelle Thibault. Votre manière d'étudier l'intérieur et les modes de vie peut-elle être dissociée de votre propre vécu ? En quoi vos propres expériences habitantes ont-elles orienté votre regard?

- Monique Eleb. Pourquoi me suis-je intéressée à l'intérieur? Je suis née à Casablanca. Enfant puis adolescente, je fréquentais des gens de tous les milieux. J'étais issue de la classe moyenne, mes camarades de classe, au lycée, appartenaient majoritairement à une bourgeoisie très élevée. La plupart d'entre eux vivaient dans des villas exceptionnelles situées dans des quartiers résidentiels très riches mais quelques-unes d'entre elles habitaient des HLM. J'ai ainsi passé mon enfance et mon adolescence dans des maisons très différentes les unes des autres. J'ai développé dès cette époque un intérêt pour les maisons en comparant des manières de vivre. Je passais mes vacances à Vichy, où mon père avait acheté un salon de coiffure en face de l'opéra. Nous vivions dans un quartier résidentiel proche du centre-ville, habité par la petite bourgeoisie, et j'y découvrais des choses que je regardais comme des curiosités ethnographiques, des personnes qui marchaient avec des patins dans leur maison... J'ai alors sans doute acquis ce goût de comprendre comment les gens vivaient et cela a été déterminant quand s'est développé mon intérêt pour l'habitat. La ville de Casablanca a indéniablement formé mon regard, comme celui de beaucoup de personnes, dont celui de I'architecte Jean-Philippe Vassal, qui m'a raconté qu'enfant, il circulait dans de nombreux domiciles avec sa mère couturière. Ces expériences sont fondamentales. J'allais également à la bibliothèque américaine située à proximité de chez moi. J'y regardais des magazines comme Good Housekeeping, à cent lieues de ce que je vivais, au sujet desquels je développais une forme de fascination ironique. Au Maroc, nous avions des domestiques, à une époque où nous n'aurions plus pu le faire en France. La figure de la ménagère et la science de l'art ménager qui étaient médiatisées dans ces revues m'étaient étrangères. J'avais cependant des voisins américains et la première chose qu'ils avaient déménagée était un énorme congélateur ; nous nous demandions à quoi cela pouvait bien servir. La femme, qui portait des rouleaux sur la tête, était une ménagère, ce que ma mère, qui travaillait, n'était pas. J'avais un attrait pour tous ces comportements que je ne connaissais pas et mes recherches ultérieures ont été marquées par ces expériences.

- Estelle Thibault. Pouvez-vous également nous parler des études de cas, qui concernent plutôt l'étude de l'habitation contemporaine?

- Monique Eleb. J'ai abordé des thématiques et des périodes très différentes et mes travaux récents le reflètent. Le fait de faire coexister la théorie et le travail de terrain a été très fécond. En 1984, je me suis demandé comment former les étudiants à un regard sociologique et à une critique de la critique architecturale - car les revues d'architecture actuelles regorgent de critiques dithyrambiques sur les derniers bâtiments construits et les innovations qu'ils sont supposés proposer. J'ai construit un enseignement qui associait des études de cas menées pendant trois mois, suivies d'une coopération avec un enseignant architecte sur un exercice 
de projet utilisant les savoirs acquis. Je choisissais chaque année un bâtiment qui avait été très médiatisé et nous allions voir comment les habitants l'avaient reçu. Mais il s'agissait également de leur faire saisir ce qu'habiter signifiait, pour les habitants. Je faisais intervenir l'architecte et éventuellement le maître d'ouvrage, qui leur parlait de la commande, des intentions, des règlements et des avatars dans le développement du projet. Après des cours de méthode, sur l'observation et sur l'entretien, sur la lecture de plan, la vingtaine d'étudiants et moi allions sur le terrain où chacun obtenait un rendez-vous avec un habitant. Ils étudiaient tout le quartier, du métro jusqu'au fond de la salle de bains, et effectuaient des relevés de plans habités, couplés à des entretiens, à l'observation et à la prise de photographies. Ces travaux d'étudiants, que j'ai conservés, constituent une documentation hors du commun. Cela formait les jeunes architectes de façon très efficace, mais cela permettait aussi d'étudier l'évolution des façons de proposer de l'habitat aux Français et comment ils s'y étaient adaptés ou non. Car il s'agissait très souvent de bâtiments très publiés, qui devenaient des modèles reproduits dans toute la France. Les étudiants étaient bons dessinateurs pour certains, et produisaient des documents permettant des comparaisons, par exemple entre les différents appartements trois-pièces d'un immeuble et leur réception par les habitants. Les entretiens montraient comment ils évaluaient les logements, ce qu'ils avaient trouvé intéressant dans les éventuelles innovations proposées. Le travail ultérieur consistait à se demander comment répondre aux critiques formulées par les habitants, et de quelle manière améliorer les dispositifs. Ces enquêtes, qui portaient le plus souvent sur des opérations de logement social, révélaient des thématiques typiquement françaises, portant par exemple sur l'absence de rangements pour le linge, la question de l'intimité, les espaces ouverts, etc.

- Estelle Thibault. L'enseignement a ainsi été un lieu essentiel de la transmission de vos travaux vers les jeunes architectes. Aujourd'hui, le corpus des études sur l'habitation s'est élargi et elles sont menées dans différents champs disciplinaires, notamment la géographie. Vos propres travaux ont également connu un écho international, par le biais de votre implication dans certaines réflexions à caractère prospectif, mais sont également prolongés, aujourd'hui, par des projets de recherche menés à l'échelle européenne. Enfin le projet d'Architectures de la vie privée n'est pas clos, puisque vous travaillez actuellement à l'écriture du troisième tome...

- Monique Eleb. La pratique pédagogique des études de cas s'est diffusée dans d'autres écoles d'architecture, la transmission s'est opérée par le biais de l'enseignement ainsi que par les jeunes docteurs que j'ai formés et qui enseignent aujourd'hui à leur tour. Ce que j'écris est en outre assez facile à lire par les étudiants, ce qui favorise l'appropriation. L'ouvrage Penser l'habiter, notamment, a eu un impact assez fort sur les projets de la jeune génération (ELEB-VIDAL, CHÂTELET, MANDOUL, 1988). Je peux également parler de l'expérience de la consultation sur le Grand Paris, débutée en 2008, pendant laquelle j'ai été associée à l'agence d'architecture et d'urbanisme néerlandaise MDRDV (Winy Maas, Jacob van Rijs et Nathalie de Vries). Ce fut une aventure exceptionnelle qui a également produit de nombreux échos.

Concernant le troisième tome d'Architectures de la vie privée auquel je travaille actuellement, il portera essentiellement sur la période de l'entre-deux-guerres. Je suis justement sur le point d'écrire le chapitre sur les femmes, dans lequel j'identifie plusieurs figures comme la garçonne, la suffragette, etc., et surtout celle de la ménagère dont les dures conditions d'existence apparaissent en lisant les traités de savoir-vivre et en observant la configuration des logements. À cette aune, on mesure l'inconfort total des maisons, des cuisines et des équipements domestiques. 
En termes de prolongement, je dois également mentionner le travail mené depuis quinze ans avec des chercheurs italiens, portugais. Il se déploie aujourd'hui au sein du réseau "European Middle Class Mass Housing » qui est une action portée par la COST (European Cooperation in Science and Technology). Ce travail collectif, coordonné par Ana Vaz Milheiro (Lisbonne) et Gaia Caramellino (Milan), vise à étudier un pan finalement relativement négligé de la production architecturale depuis les années 1950. Je suis intervenue récemment, pour conclure le dernier colloque, par une communication intitulée « Enseigner la vraie maison en France au XXe siècle » (8 et 9 mars 2021). Enfin mon dernier livre, coécrit avec Lionel Engrand, porte sur l'architecture des maisons individuelles : nous y explorons les raisons pour lesquelles les Français ont simultanément plébiscité la modernisation des équipements intérieurs et majoritairement préféré une écriture traditionnelle de l'enveloppe, qui reste dominante dans l'essentiel de la production actuelle (ELEB, ENGRAND, 2020). 


\section{NOTES}

1. Nicole Eleb-Harlé (1943-2014), architecte urbaniste et enseignante chercheuse.

2. Tony Côme, L'Institut de l'environnement : une école décloisonnée. Urbanisme, architecture, design, communication, Paris, B 42, 2017.

3. Christian Gaillard et al., Sciences humaines et environnement. Orientations bibliographiques, Paris, Institut de l'environnement, 1971.

4. Christian Gaillard et al., L'Architecte, lui-même et les autres, Grenoble, Presses universitaires de Grenoble, 1973.

5. Elle s'est ensuite orientée vers l'anthropologie, voir Christelle Robin (dir.) Espaces des autres. Lectures anthropologiques d'architecture, Paris, La Villette, 1987.

6. Ou UP1, première dénomination de l'école d'architecture de Paris Villemin, créée en 1969, par suite de la fin de la section architecture de l'École nationale supérieure de beaux-arts.

7. Michèle Huguet, Les Femmes dans les grands ensembles: de la représentation à la mise en scène, Paris, CNRS, 1971.

8. Paul-Henri et Marie-José Chombart de Lauwe et al., Famille et habitation, t. I. Sciences humaines et conceptions de l'habitation, Paris, CNRS, 1960.

9. Colette Pétonnet, Ces gens-là, Paris, Maspéro, 1968.

10. Henri et Marie-Geneviève Raymond et al., L'Habitat pavillonnaire, Paris, L'Harmattan, 1966.

11. Aldo Rossi, Scientific Autobiography, Cambridge, Mass., The MIT Press, 1981.

12. Pierre Bourdieu, Esquisse d'une théorie de la pratique, Genève, Librairie Droz, 1972.

13. Marcel Mauss, "Les techniques du corps » (1934), dans Sociologie et Anthropologie, Paris, Presses universitaires de France, 1968.

14. Erving Goffman, La Mise en scène de la vie quotidienne, t. I. La Présentation de soi (1959) et t. II. Les Relations en public (1971), Paris, Minuit, 1973 et Erving Goffman, Les Rites d'interaction (1967), Paris, Minuit, 1974.

15. Gwendolyn Wright, Moralism and the Model Home: Domestic Architecture and Cultural Conflict in Chicago, 1873-1913, Chicago / Londres, University of Chicago Press, 1980.

16. Donatella Calabi, Architettura domestica in Gran Bretagna, 1890-1939, Milan, Electa, 1982.

17. Pierre Le Muet, Maniere de bastir pour toutes sortes de personnes..., Paris, M. Tavernier, 1623.

18. "La relation objet-figuration nous introduit dans la manière de penser de toute une époque ». Pierre Francastel, La Figure et le Lieu, Paris, Gallimard, 1967, p. 75.

19. Philippe Ariès, Georges Duby, Michelle Perrot (dir.), Histoire de la vie privée, t. IV. De la Révolution à la grande guerre, Paris, Seuil, 1987.

20. Lion Murard, Patrick Zylberman, Le Petit Travailleur infatigable. Villes-usines, habitat et intimités au XIX siècle, Paris, Recherches, 1980.
21. Norbert Elias, La Société de cour (1969), Paris, Flammarion, 1974.

22. Ibid., p. 26-27.

23. Sigmund Freud, Essais de psychanalyse (1915-1923), Paris, Payot, 2001.

24. Le Programme d'architecture nouvelle a été créé en 1972 par le Plan construction de façon à améliorer la qualité architecturale. La première édition visait à favoriser l'innovation dans un contexte de logement social alors fortement stigmatisé par la production en série.

25. Chombart de Lauwe et al., 1960, cité n. 8

26. Eugène Viollet-le-Duc, Histoire d'une maison, Paris, J. Hetzel, 1873.

\section{BIBLIOGRAPHIE SÉLECTIVE}

- GAILLARD, NICK, VIDAL, LÉVY-GRANGE, 1973 : Christian Gaillard, Philippe Nick, Monique Vidal, Michèle LévyGrangé, L'Architecte, lui-même et les autres, Grenoble, Presses universitaires de Grenoble, 1973.

- GAILlaRD, Teboul, VIDAL, 1973 : Christian Gaillard, Michèle Teboul, Monique Vidal (dir.), Espaces des sciences humaines, Questions d'enseignement en architecture, Paris, Éditions médicales et universitaires, 1973.

- ELEB-VIDAL, 1980 : Monique Eleb-Vidal, « Se construire et habiter. Proposition d'analyse psycho-sociale clinique ", thèse de doctorat, université Paris VII, 1980. - ELEB-VIDAL, 1987 : Monique Eleb-Vidal, «Inculcation corporelle et appartenance sociale dans le logement ", dans Christelle Robin (dir.), Espaces des autres, Lectures anthropologiques d'architecture, La Villette, 1987, p. 189-195.

- ELEB-VIDAL, CHÂTELET, MANDOUL, 1988 : Monique Eleb-Vidal, Anne-Marie Châtelet, Thierry Mandoul, Penser l'habité, le logement en questions, Liège, Mardaga, 1988.

- Eleb-VIDAL, avec DebarRe-BlanChard, 1989 : Monique Eleb-Vidal, avec Anne Debarre-Blanchard, Architectures de la vie privée. Maisons et mentalités, XVII ${ }^{e}$-XIX siècles, Bruxelles / Paris, Archives d'architecture moderne / Hazan, 1989.

- ELEB, 1994 : Monique Eleb, L'apprentissage du « chezsoi ». Le groupe des maisons ouvrières, Paris, avenue Daumesnil, 1908, Marseille, Parenthèses, 1994.

- ElEB, avec DeBARRE, 1995 : Monique Eleb, avec Anne Debarre, L'Invention de l'habitation moderne, Paris 1880-1914. Architectures de la vie privée, suite, Bruxelles / Paris, Archives d'architecture moderne / Hazan, 1995. - EleB, ChÂTElET, 1998 : Monique Eleb, Anne-Marie Châtelet, Urbanité, sociabilité et intimité. Des logements d'aujourd'hui, Paris, L'Épure, 1998.

- ElEB, VIOlEAU, 2000 : Monique Eleb et Jean-Louis Violeau, Entre voisins. Dispositif architectural et mixité sociale, Paris, L'Épure, 2000.

- ELEB, 2002 : Monique Eleb, «Généalogie de l'habitation et histoire sociale ", Les Cahiers de la recherche architecturale et urbaine, $\mathrm{n}^{\circ}$ 9/10, 2002, p. 137-150.

- Eleb, Bendimérad, 2011 : Monique Eleb, Sabri 
Bendimérad, Vu de l'intérieur. Habiter un immeuble en Île-de-France, 1945-2010, cat. exp. (Paris, Maison de l'architecture d'île-de-France), Paris, Archibook + Sautereau, 2011.

- ELEB, SIMON, 2013 : Monique Eleb, Philippe Simon, Le Logement contemporain : entre confort, désir et normes, 1995-2012, Bruxelles, Mardaga, 2013.

- ELEB, 2015 : Monique Eleb, Les 101 mots de l'habitat à l'usage de tous, Paris, Archibooks, 2015.

- Eleb, BendimÉRAD, 2018 : Monique Eleb, Sabri Bendimérad, Ensemble et séparément : des lieux pour cohabiter, Bruxelles, Mardaga, 2018.

- ELEB, COHEN, 2019 : Monique Eleb, Jean-Louis Cohen, Casablanca: mythes et figures d'une aventure urbaine, [Paris], Hazan, 1998.

- ELEB, ENGRAND, 2020 : Monique Eleb, Lionel Engrand, La Maison des Français : discours, imaginaires, modèles (1918-1970), Bruxelles, Mardaga, 2020. 